Copyright ( 2020 University of Bucharest Printed in Romania. All rights reserved

ISSN print: $1224-5984$

ISSN online: $2248-3942$
Rom Biotechnol Lett. 2020; 25(2): 1432-1439

doi: $10.25083 / \mathrm{rbl} / 25.2 / 1432.1439$

Received for publication, December, 21, 2017

Accepted, August, 6, 2018

Original paper

\title{
Antioxidant effect of a vegetal grape waste complex, demonstrated in relevant dermal and epidermal cellular systems
}

\author{
LUIZA M. CRĂCIUN ${ }^{1,2,3}$, BRANDUSA G. DUMITRIU ${ }^{1,3}$, LAURA OLARIU ${ }^{1,3}$, \\ DIANA M. ENE ${ }^{1}$, ABDI ADIL ${ }^{2,3}$, NATALIA ROSOIU ${ }^{1,2,3}$ \\ ${ }^{1}$ SC Biotehnos SA, 3-5 Gorunului Street, 075100-Otopeni, Ilfov, Romania \\ ${ }^{2}$ The Doctoral School of Applied Sciences, Ovidius University, Constanta, Romania \\ ${ }^{3}$ Academy of Romanian Scientists, 54 Splaiul Independentei 050094, Bucharest, Romania
}

\begin{abstract}
The complete capitalisation of vegetal raw materials as renewable natural resources is a current study direction that is based on the diversification of active compounds identified and isolated from certain sources, the effectiveness of extracting technologies and the definition of a relevant spectrum of biological action.

The study is focused on highlighting the antioxidant effect induced by components of winemaking waste (TES extract) in dermo-epidermal cells. The tests was performed on normal fibroblast and normal human keratinocytic cell lines, preceded by acellular screening for DPPH free radical reduction and total antioxidant capacity assessment. At the cellular level, intracellular oxygenated oxygen (superoxide and hydrogen peroxide) and intracellular glutathione were monitored by flow cytometry, comparing with the intracellular antioxidant enzyme activity (Superoxide dismutase ant Catalase). The results confirm a concerted antioxidant effect for TES, suggesting many pharmaceutical and cosmetic applications.
\end{abstract}

Keywords TES extract, DPPH, Total Antioxidant Status, SOD, Catalase, Superoxide anion, Hydrogen peroxide.

To cite this article: CRĂCIUN LM, DUMITRIU BG, OLARIU L, ENE DM, ADIL A, ROSOIU N. Antioxidant effect of a vegetal grape waste complex, demonstrated in relevant dermal and epidermal cellular systems. Rom Biotechnol Lett. 2020; 25(2): 1432-1439. DOI: $10.25083 / \mathrm{rbl} / 25.2 / 1432.1439$

*Corresponding author: LAURA OLARIU, SC Biotehnos SA, 3-5 Gorunului Street, 075100-Otopeni, Ilfov, Romania, Tel.: 031/710.24.02; 031/710.23.82. Fax: 031/710.24.00

E-mail: lolariu@biotehnos.com 


\section{Introduction}

Oxidative stress results from the increased generation of ROS/ RNS or from a decrease in protective antioxidant ability, being characterized by the reduced capacity of endogenous systems to fight against direct attack on target biomolecules (HUSSEIN et al [1], PISOSCHI et al [2]). ROS and related species are mediators of the inflammatory response. Instead, ROS also activates cellular signaling, increasing the production and release of proinflammatory cytokines, thus perpetuating the inflammatory response. Well-controlled inflammatory responses are part of the innate immunity of our body, protecting against invading pathogens (ARUOMA et al [3]). ROS and inflammation are closely related, so many enzymes or antioxidant proteins, as well as non-protein antioxidants, have anti-inflammatory activities (ZHOU et al [4]). Epidemiological studies have demonstrated the ability of antioxidants to reduce the effects of reactive oxygen species and to decrease the incidence of cancer and other degenerative diseases. The first types of antioxidant defense systems developed against oxidative lesions are those that prevent the appearance of ROS and those that block or capture, the radicals that are formed. These systems present in aqueous solutions and membrane cell compartments can be enzymatic and non-enzymatic. The reparative cellular process represents another important antioxidant system which eliminates damaged biomolecules (RINNERTHALER et al [5], DUMITRIU et al [6]). Besides the catabolic effects in the chain that reactive species produce in the body, the oxidative degradation occurs also in the food, causing organoleptic changes in the nutritional intake. In this context, the use of natural antioxidants as adjuvant in food can reach two goals: prevention/ therapeutics by blocking cellular oxidative stress, respectively preserving the nutritional properties and the appearance of the preparations with the prolongation of the shelf life. A significant aspect is the replacement of synthesis compounds with natural complexes, with the exclusion of associated harmful effects, with beneficial effects on the body (DEAVALL et al [7], DUMITRIU et al [8]). The aim of this study is to determine the effect of active extracts from grape waste on some reactive species of oxygen, inorganic radical species: superoxide $\left(\mathrm{O}^{\circ}{ }^{-}\right)$and non-radical: hydrogen peroxide $\left(\mathrm{H}_{2} \mathrm{O}_{2}\right)$, and antioxidant enzymes superoxide dismutase (SOD)-catalase (CAT).

\section{Materials and Methods}

\section{Materials}

Vegetable material: grape marc, the residue obtained from the grapes processing in the wine industry and will be referred to as the TES extract. After 7 days of fermentation, the plant material was dehydrated in a vacuum oven at $40^{\circ} \mathrm{C}$ and then ground. The TES extract was solubilized in water, $70 \%$ ethanol or $70 \%$ methanol in a concentration of $50 \mathrm{mg} / \mathrm{ml}$ and $100 \mathrm{mg} / \mathrm{ml}$ ground TES extract. Alcoholic extracts have been used in tests to determine total antioxidant capacity and to determine antiradical activity in the acellular system.

Standardized cell lines: Fibroblast (normal cell line HS27) - cells with high proliferative capacity, cell cultures reach confluency relatively quickly $(80 \%$ confluence in 3 days). Cells were cultured in monolayer in DMEM (Dulbecco's Modified Eagle's Medium / Nutrient Mixture F-12 Ham, code: D8437, Sigma-Aldrich) supplemented with $10 \%$ fetal bovine serum (code: F7524, Sigma-Aldrich) and 1\% Antibiotic Antimycotic Solution (100×) (Cod: A5955, Sigma Aldrich) used in tests between passages 20-40. Keratinocit (HaCaT) - epithelial squamous cell with a remarkable regenerative potential, which undergoes a differentiation process during its migration from the level of the germinative layer to the cortical layer, descuamative. The cells were cultured in Dulbecco's Modified Eagle's Medium (DMEM) (code: 30-2002, ATCC®) supplemented with $10 \%$ fetal bovine serum, $1 \%$ Antibiotic Antimycotic Solution (100×) (Cod: A5955, Sigma Aldrich) and used in experiments at passages 40-50.

Chemical Substances: The control agent used, were N-acetyl-cysteine (code: SC-202232, Santa Cruz Biotechnology) as antioxidant (ESPOSITO et al [9] and DeCHATELET et al [10]). For total antioxidant capacity (TAS) - TAS (total antioxidant status) kit - RANDOX Laboratories Ltd., UK. (Code: NX2332) was used. Catalase from bovine erythrocytes (code: C1345), Superoxide dismutase (code: 9697), hydrogen peroxide (code: 216763), EDTA (code: E1644), alpha-diphenyl- $\beta$-picrylhydrazyl radical (DPPH; code: D9132), Xanthine (code: X0626), Xanthine oxidase (code: X1875), Cytocrome C (code: C7752) were purchased from Sigma.The analysis of reactive oxygen species was performed by flow cytometry (FACS Canto II) using fluorescence markers HE (dihydroetidine) and DCFH-DA (2', 7'-dichlorofluorescein diacetate).

\section{Methods}

a) The DPPH (2,2-difenyl-1-picrylhydrazyl) radical reduction method is a spectrophotometric method and was introduced by M. BLOIS [11] and developed and improved by BONDET et al [12]; BRAND-WILLIAMS et al [13]. DPPH Free Capability Analysis is a colorimetric test that measures the ability of antioxidants to react directly with DPPH (scavenge) radicals by spectrophotometric monitoring of its absorption at $517 \mathrm{~nm}$. The ability of the compounds to scavenge DPPH * is determined by their ability to yield electrons or hydrogen, that is to say the magnitude of the oxidoreduction potential of the antioxidants studied. Antiradical activity (ARA) was 
defined as the amount of antioxidant needed to decrease the initial DPPH * concentration by $50 \%$ and represents the effective concentration, $\mathrm{EC}_{50}$.

b) Evaluation of total antioxidant status (TAS) reduction of ABTS (2,2'-azinobis (3-ethylbenzothiazoline6-sulfonic acid) radical, indicating the level of antioxidants in the tested extract. Reducing the blue-green ABTS solution by hydrogen donation by the antioxidant is quantified by measuring the absorption spectrum at the characteristic wavelength $(405 \mathrm{~nm})$. In the colorimetric reaction to determine $\mathrm{ABTS}$, metmioglobin reacts with hydrogen peroxide to produce the ferilmioglobin radical. This radical oxidizes the ABTS molecule to $\mathrm{ABTS}^{+}$ with its characteristic color. Antioxidants inhibit color development because they reduce $\mathrm{ABTS}^{+}$, (EREL [14] KAMPA et al [15]).

c) Determination of the enzymatic activity of bovine liver catalase and intracellular catalase

Catalase exerts a dual action because it catalyzes the decomposition of $\mathrm{H}_{2} \mathrm{O}_{2}$ with $\mathrm{H}_{2} \mathrm{O}$ and $\mathrm{O}_{2}$ formation (catalase activity) and on the other hand favors the oxidation of hydrogen donors (methanol, ethanol, formic acid, phenols) with consumption of one mole of peroxide (peroxidase activity) (RINNERTHALER et al [5]). The decomposition of hydrogen peroxide is a first-order reaction, the rate of which is always proportional to the peroxide concentration. Therefore, in order to avoid a rapid decrease in the initial reaction rate, determinations are made at relatively low $\mathrm{H}_{2} \mathrm{O}_{2}$ concentrations (about $0.01 \mathrm{~mol} / \mathrm{l}$ ) (MUELLER et al [16].

d) Determination of the enzymatic activity of superoxide dismutase from bovine erythrocytes and intracellular superoxide dismutase

In order to evaluate the enzymatic activity of SOD, an indirect method was used by coupling the superoxide anion decomposition reaction in the presence of SOD with the cytochrome $\mathrm{c}$ reduction reaction by the superoxide radical (an enzymatic product in the reaction between xanthine and $\mathrm{O}_{2}$ ). An enzymatic unit will decrease the rate of cytocrome c reduction by $50 \%$ in a Xanthine/ Xanthineoxidase coupled system. SOD activity was measured by inhibiting the cytochrome $\mathrm{c}$ reduction by the superoxide anion through the Xantin/Xantin-oxidase system, and spectrophotometrically monitoring the absorbance change at $550 \mathrm{~nm}$ (McCORD et al [17]).

e) Evaluation of cellular glutathione content by flow cytometry

Glutathione (GSH) is a tripeptide sulfhydryl (glucys-gly) present in millimolar concentrations in most eukaryotic cells. It is an intracellular reducing agent involved in multiple cellular processes, protecting the cell from free radical species, hydrogen peroxide and organic peroxides, catalysed glutathione-S-transferase and glutathione peroxidase reactions. Glutathione has a double role: in detoxification processes by binding to heavy metals, solvents, pesticides and their transformation into excretable substances and as an antioxidant by keeping the -SH groups of proteins in reduced form. His detection is important in estimating intrinsic antioxidant cellular status. Determination of intracellular glutathione will be done by labeling with fluorescent antibodies (e.g. ABCAM anti-glutathione antibody, An Alexa-Fluor 488 conjugated goat anti-mouse $\operatorname{IgG}(\mathrm{H}+\mathrm{L})$ and flow cytometry assay (HEDLEY et al [18]).

f) Evaluation of intracellular oxidative stress by flow cytometry by simultaneous identification of intracellular oxygenated radicals (superoxide and hydrogen peroxide)

The ability to reduce the $\mathrm{H}_{2} \mathrm{O}_{2}$ and $\mathrm{O}_{2}$-intracellular level of a test substance is assessed at the cellular level to detect the possible effect on defense processes against the harmful effects of reactive oxygen species. The superoxide anion is formed by the electron transport chain, NADPH oxidases, nitric oxide synthase, xanthine-oxidase and cytochrome P-450, depending on pathological and physiological stimuli, being the precursor of hydrogen peroxide, peroxynitrite, and other strongly oxidized species that produce in small amounts modulates enzymatic activity and signal transduction cascades, but in large amounts produces cellular oxidative stress (QUIJANO et al [19]). The method of simultaneous identification of $\mathrm{H}_{2} \mathrm{O}_{2}$ and $\mathrm{O}_{2}$ intracellular levels - by marking with DCFH-DA or HE: DCFH-DA is incorporated into the hydrophobic lipid region where the hydrolytic enzymes cleave the remaining diacetate, leaving the DCFH (dichlorofluorescein) nonfluorescent molecule to enter the cytoplasm due to polarity. Under cell activation conditions, hydrogen peroxide and intracellular peroxidases oxidize the molecule to DCF - fluorescent compound emitting at 530nm (FITC-A). Hydroxyethidine (HE) permeable through the cell membrane, forming after oxidation by superoxide anions the ethidium bromide binds to nucleic acids and emitting at $620 \mathrm{~nm}$ (PE-A) (CARINI et al [20]; ROBINSON et al [21]).

\section{Results and Disscusions}

The antioxidant/antiradical action in vitro in the acellular system was determined by two spectrophotometric methods:

DPPH assay: DPPH-scavenging capability is determined by measuring the absorbance (after being kept 30 minutes, dark) at the wavelength $\lambda=517 \mathrm{~nm}$ (maximum DPPH absorption) that is proportional to the free radical concentration of solution. 

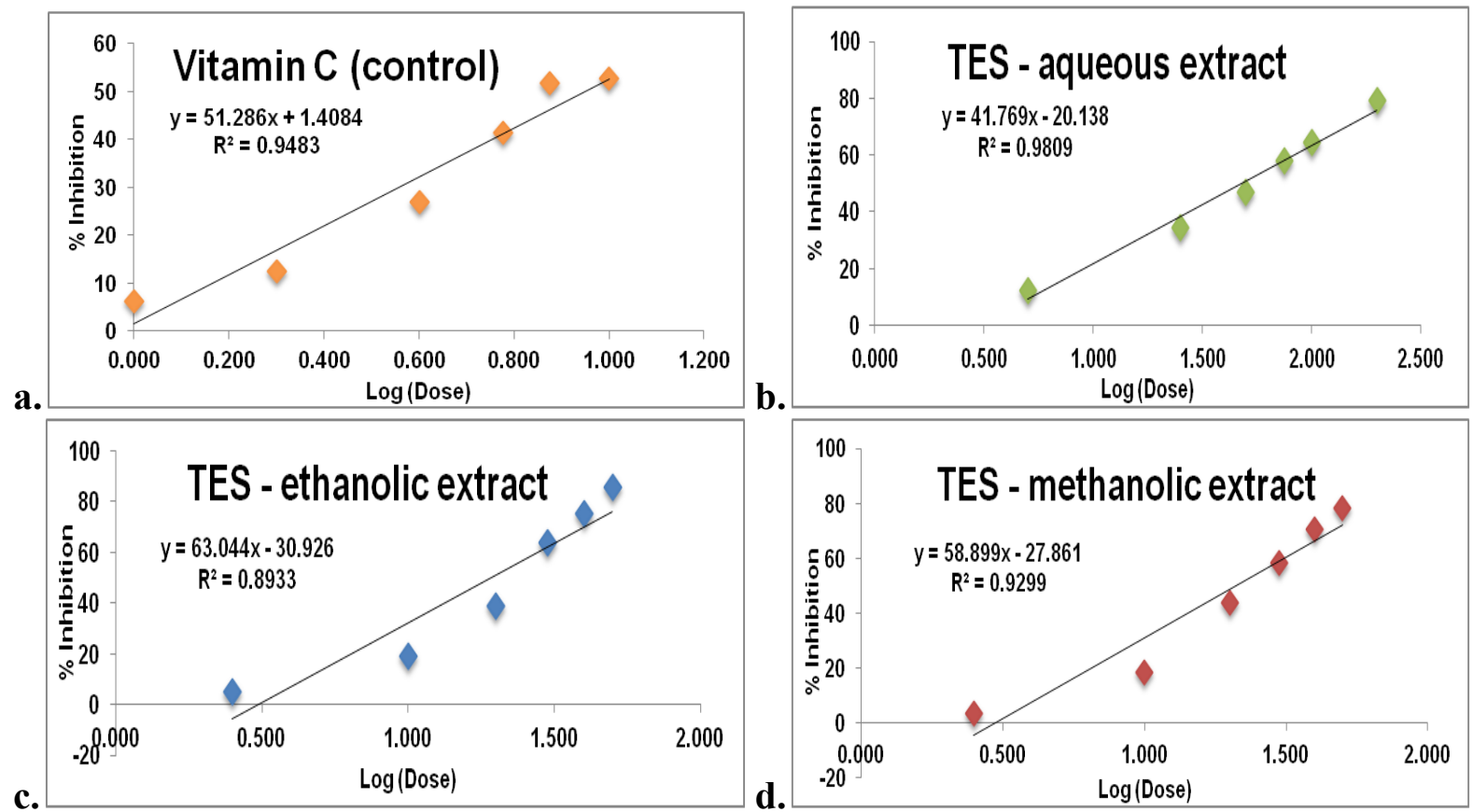

Figure 1. Reduction of DPPH in the presence of TES extract: Three variants of TES extract were prepared in different solubilizing media, namely water, $70 \%$ ethanol and $70 \%$ methanol. A solution of $1 \mathrm{mg} / \mathrm{ml}$ Vitamin C in water was used as reference. For each sample, a blank consisting of methanol, distilled water and the mixture of interest is made. The graphically plotted results are expressed as percent inhibition of DPPH free according to the applied dose as follows: a. Vitamin C (1 mg/ ml); b. Aqueous extract (50 mg/ ml); c. $70 \%$ ethanolic extract (50 mg/ ml); d. $70 \%$ methanolic extract $(50 \mathrm{mg} / \mathrm{ml})$.

Total Antioxidant Status (TAS) assay: was performed with the TAS (Total Antioxidant Status) kit - RANDOX Laboratories Ltd., UK, providing a colorimetric method of antioxidant levels in the unknown sample. The ABTS test measures the ability of an antioxidant to capture ABTS ions generated in aqueous solution, compared to standard Trolox (water-soluble vitamin E analog). The ABTS radical is generated by the reaction of a strong oxidizing agent with ABTS salt.

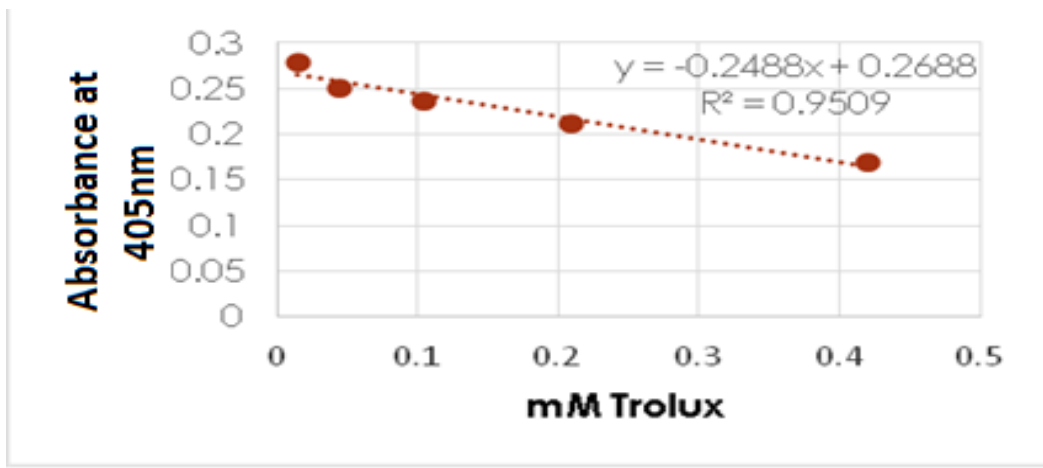

Figure 2. Calibration Curve with Trolux (Vitamin E Synthetic)

Table 1. Evaluation of total antioxidant concentration and antioxidant/antiradical effect

\begin{tabular}{ccc}
\hline Sample & $\begin{array}{c}\text { DPPH } \\
\mathbf{E C}_{\mathbf{5 0}}(\boldsymbol{\mu} \mathbf{l} \text { extract } / \mathbf{~ m l})\end{array}$ & $\begin{array}{c}\text { TAS } \\
\mathbf{m o l} / \mathbf{m l}\end{array}$ \\
\hline \hline TES aqueous extract & 48 & 112,76 \\
\hline TES ethanolic extract & 19 & 282,11 \\
\hline TES methanolic extract & 21 & 268,03 \\
\hline Vitamin C & 10 & - \\
\hline
\end{tabular}


The data analysis in the table above (Table 1) indicates that the ethanolic TES extract has a higher antiradical capacity (the amount of extract required to reduce by $50 \%$ the initial DPPH concentration $\left(\mathrm{EC}_{50}\right)$ is $19 \mu \mathrm{l})$ compared to the TES extract in the other two solubilization media (water (48 $\mu \mathrm{l}$ extract) and $70 \%$ methanol $(21 \mu 1$ extract respectively).

These results correlate with those obtained in the TAS assay, which indicates that the highest amount of antioxidants is found in the ethanolic TES extract $(282.11 \mathrm{~mol} / \mathrm{ml})$.
The evaluation of the antioxidant effect of the extract on the two phase I oxidative enzymes involved in the reduction of the oxidative stress: catalase - superoxide dismutase was performed by two spectrophotometric methods, both in the in vitro (acellular) and cellular systems:

Determination of enzymatic activity of bovine liver catalase.
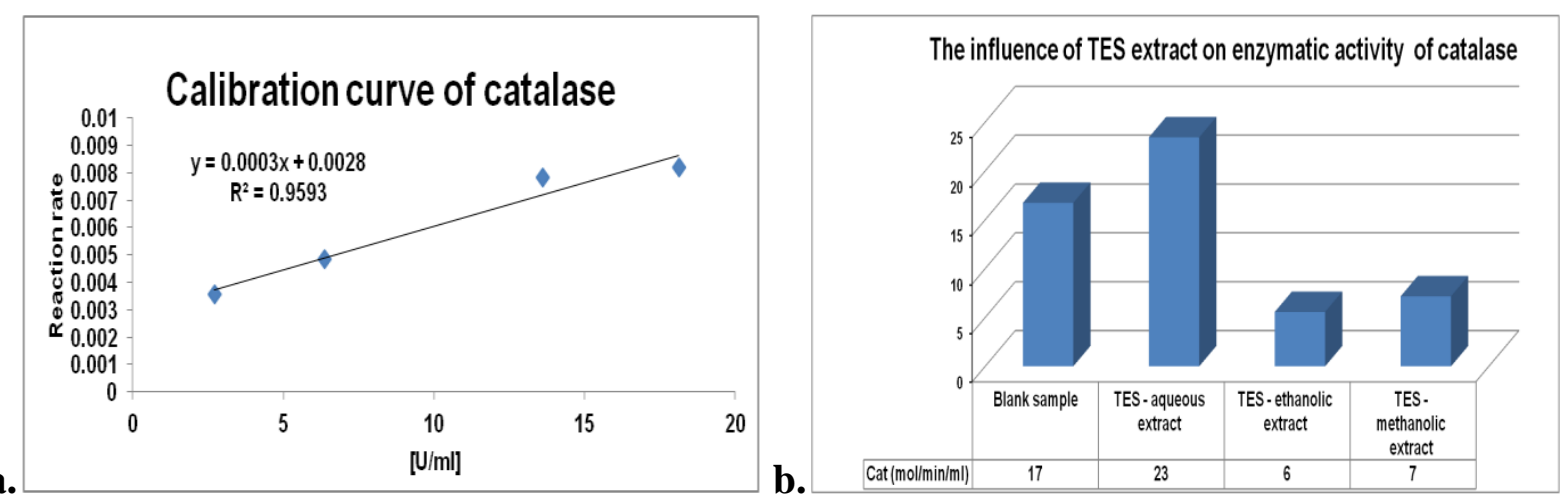

Figure 3. The effect of the TES extract on the enzymatic activity of catalase in the acellular system: a. Calibration curve of Catalase; b. Enzimatic activity of catalase in presence of $10 \mu 1$ TES extract.

The TES aqueous extract significantly increases the activity of the catalase by $40 \%$ compared to the control, thereby leading to the removal of hydrogen peroxide in the system. Due to the presence of the alcohol in the reaction medium, we have a false negative effect because it acts on the quaternary structure of the enzyme and leads to their denaturation.

Determination of the enzymatic activity of superoxide dismutase from bovine erythrocytes.

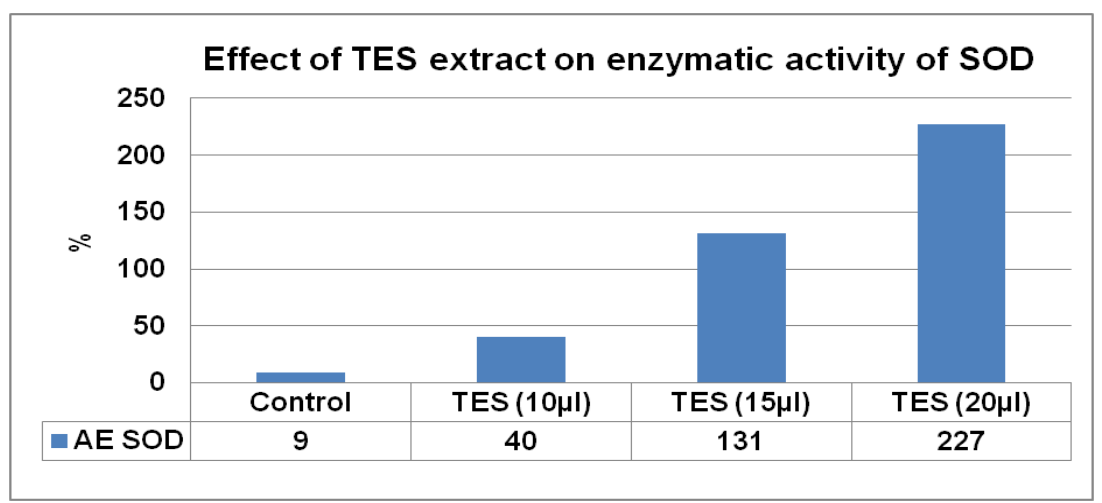

Figure 4. Variation of the enzymatic activity of SOD in the presence of TES extract depending on the applied dose.

The TES aqueous extract shows an increase in the enzymatic activity of SOD, depending on the applied dose ( $20 \mu \mathrm{l}$ extract increases by up to $227 \%$ AE SOD). Thus we can say that TES contains a compound with SOD activating action. These results confirm the antioxidant potential of the tested extract, helping to maintain the physiological redox balance by reducing oxidative stress.

Evaluation of the enzymatic activity of intracellular enzymes (Catalase, Superoxide dismutase) in the presence of TES extract:
The enzymatic activity of the major antioxidant enzymes: catalase and superoxide-dismutase was evaluated from normal human keratinocyte and fibroblasts lysates, grown under the following experimental conditions: $24 \mathrm{~h}$ adhesion, $48 \mathrm{~h}$ treatment, and stimulation with TNF $15 \mathrm{ng} / \mathrm{ml}$ and PMA $0.1 \mu \mathrm{M}$. The results are presented in the graphs below as percentages of variation of the absorbances corresponding to the enzymatic activity of the treated samples versus the untreated cell reference. 

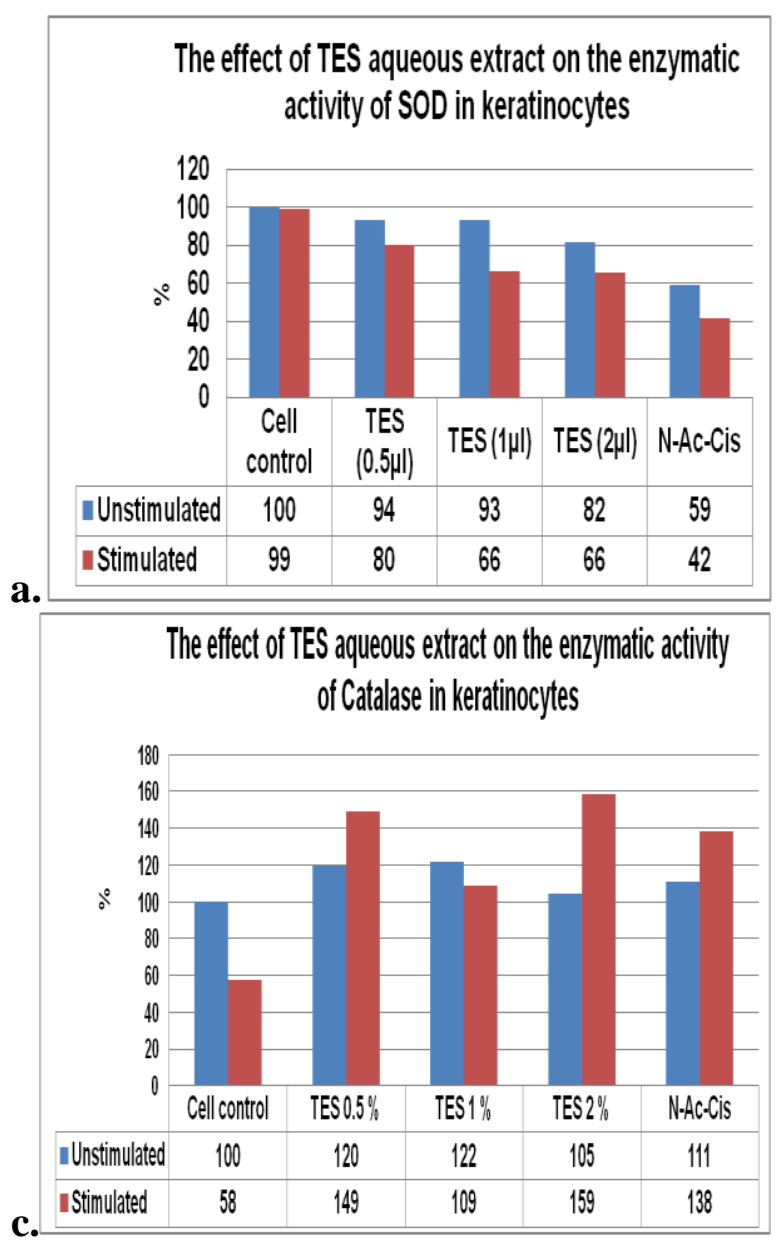

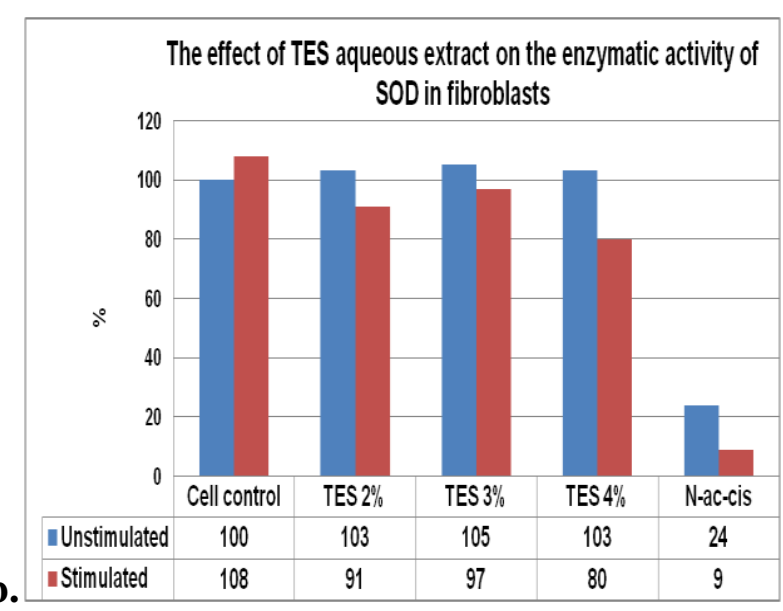

b.

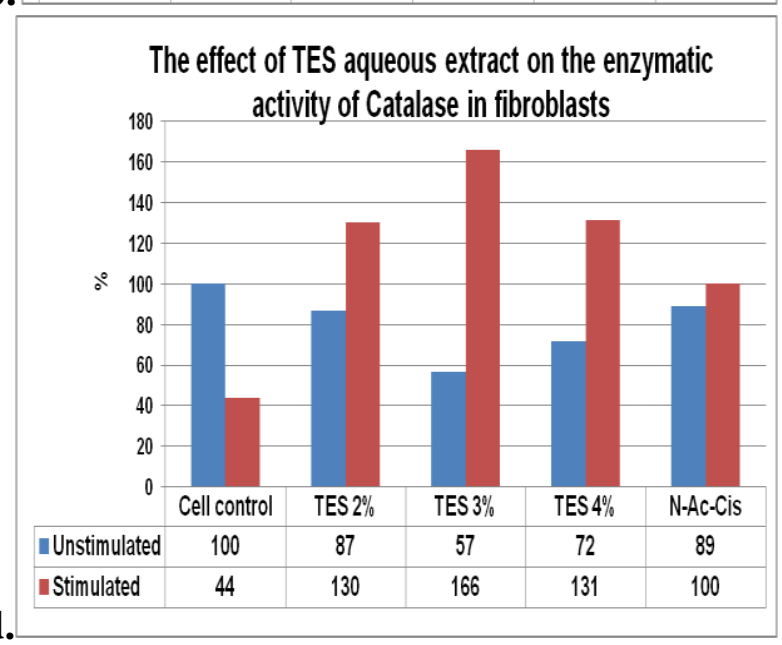

Figure 5. Influence of the TES complex on the enzymatic activity of SOD (a. HaCaT cell line; b. HS27 cell line); Influence of TES complex on enzymatic activity of catalase (c. HaCaT cell line; d. HS27 cell line).

In the case of keratinocytes treated with TES extract, there was a decrease in the enzymatic activity of SOD, in a dose-dependent manner, both in a basic metabolic status and in pro-oxidative stimulation conditions. In the case of fibroblasts treated with TES aqueous extract, the same tendency of SOD enzymatic activity decreasing is observed.

As a result of the increased enzymatic activity of endogenous catalase, both at the basal level and under oxidative stimulation conditions, the hydrogen peroxide will decrease and TES will manifest antioxidant effect.

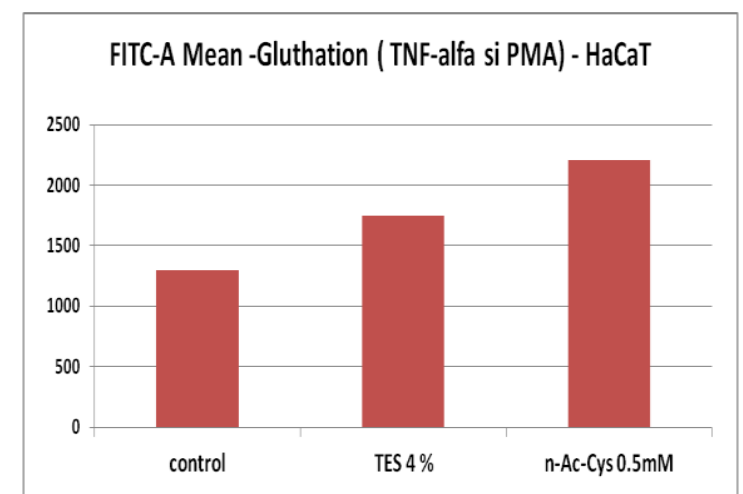

a.

Figure 6. Variation of TES extract-induced intracellular glutathione in HaCaT (a) and HS-27 cell lines

(b) pro-inflammatory stimulated.
Evaluation of cellular glutathione content by flow cytometry

Stimulation of the intrinsic antioxidant system to induce cellular protection against pro-oxidative aggression was studied on both types of dermo-epidermal cells under proinflammatory stimulation conditions with TNF $\alpha 15 \mathrm{ng} /$ $\mathrm{ml}$ and PMA $0.1 \mu \mathrm{M}$. The results are presented in the graphs below. Fluorescence intensity FITC-A is proportional to the amount of intracellular glutathione.

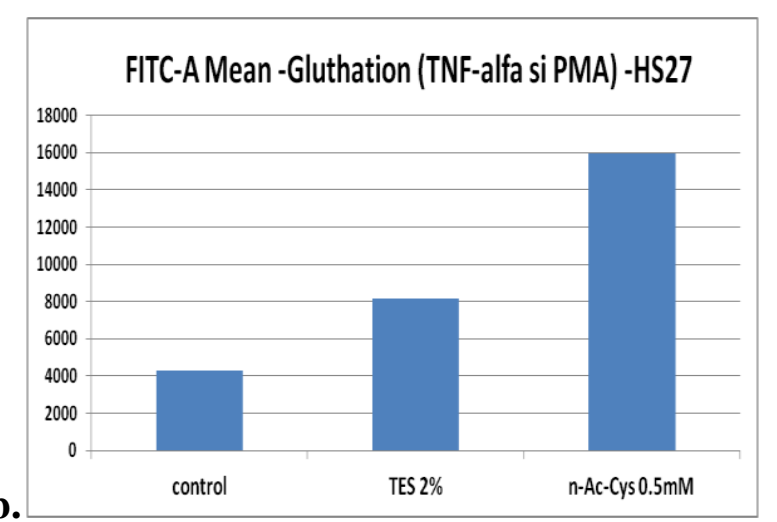


The TES complex induces an increase in cellular glutathione in both cell lines in the same way as the positive $\mathrm{N}$-acetyl-Cysteine control, thereby demonstrating its implication in the intrinsic antioxidant protective mechanisms of the cells.

Evaluation of intracellular oxidative stress by flow cytometry

The results are aquired with the FACS CantoII flow cytometer and analyzed with the Diva 6 software. The quantity of oxygen peroxide and the intracellular superoxide anion correspond to the fluorescence channel fluctuation variation

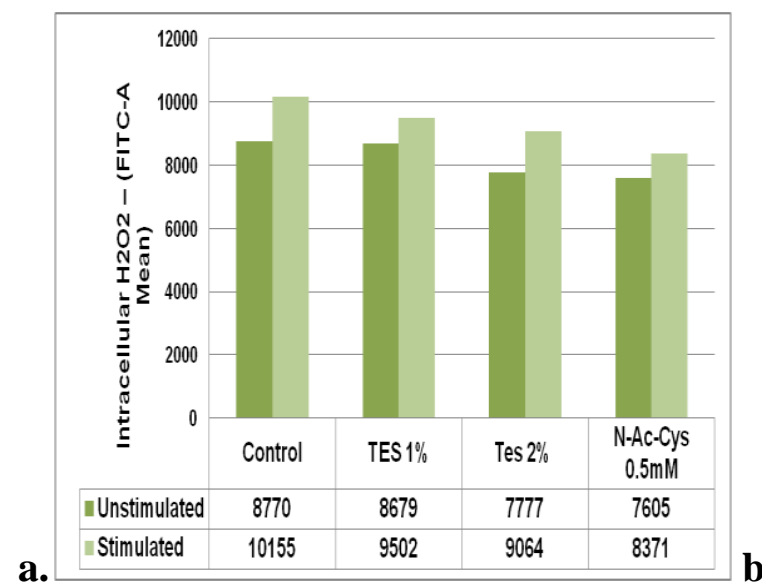

in the two coordinates: FITC - A mean - for hydrogen peroxide and PE-A mean - for the superoxide anion.

Two experimental series were performed: $48 \mathrm{~h}$ treatment under normal developmental conditions (basal level of hydrogen peroxide, in the presence and absence of the investigated compounds) and in a non-specific inflammation model induced by TNF- $\alpha+$ PMA treatment $(24 \mathrm{~h}$ pretreatment with extract $+24 \mathrm{~h}$ stimulation).

$\mathrm{N}$-acetyl-Cysteine, a precursor of L-cysteine, was used as a positive control, which acts as an intrinsic antioxidant.

The results are presented in the graphs below:

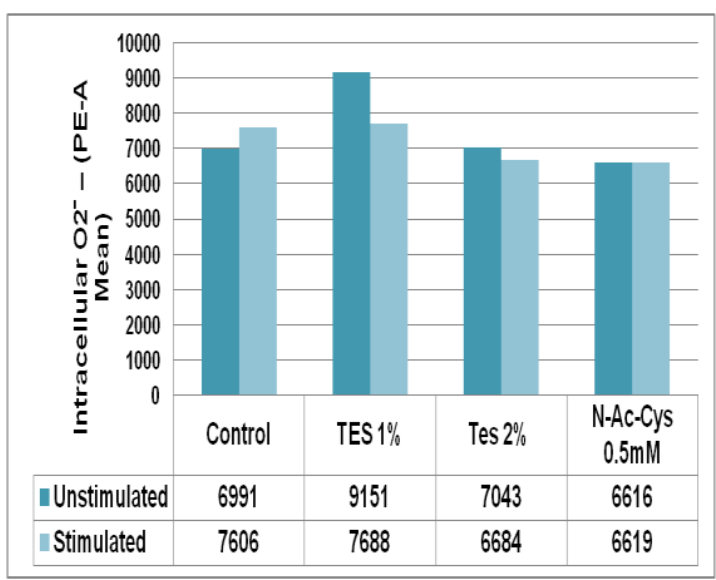

Figure 7. Effect of TES extract on $\mathrm{H}_{2} \mathrm{O}_{2}$ (a) and $\mathrm{O}_{2}^{-}$(b) in keratinocyte (HaCaT) unstimulated / stimulated with TNF- $\alpha+$ PMA.

TES aqueous extract reduces the formation of both intracellular oxygenated radicals, in unstimulated cells and also in proinflammatory and pro-oxidative stimulated cells. This action reveals its convergent antioxidant capacity, sustained also through the enzymatic system modulation.

\section{Conclusions}

The specific biological activities studies have shown an antiradical/antioxidant effect by reducing DPPH correlated with the amount of antioxidants recovered in the ethanolic extract.

The aqueous TES extract increases the enzymatic activity of catalase and SOD in acellular systems, confirming the antioxidant potential by the action of the two enzymes involved in the reduction of oxidative stress.

The acellular actions of TES extract are reflected in dermo-epidermic systems (keratinocytes and fibroblasts) through the modulation of the folowing parameters:

- decrease of hydrogen peroxide, as a result of the increased enzymatic activity of endogenous catalase (HS27 and HaCaT cell lines).

- intracellular reduction of the main reactive oxygen species $\left(\mathrm{H}_{2} \mathrm{O}_{2}\right.$ and $\left.\mathrm{O}_{2}^{-}\right)$in pro-inflammatory stimulated keratinocytes, according with enzymatic activity changes of catalase and superoxide-dismutase.

- cellular glutathione activation in fibroblasts and keratinocytes, similar with N-Acetyl -Cysteine.
This complete antioxidant screening prove multifactorial action of the grape mark extract, leading to its future capitalization in pharmaceutical and cosmetic products. The waste resulting from the winemaking process is a convenient source of antioxidant substances, considering the simplicity of the technological processes and the complex panel of antioxidant properties.

\section{Acknowledgegments}

The study was performed as part of the AOSR project: "Capitalization in industry of residues from winemaking as food additives and antioxidants".

\section{Conflict of interest}

The authors declare that they have no conflict of interest.

\section{References}

1. M.A. HUSSEIN, A Convenient Mechanism for the Free Radical Scavenging Activity of Resveratrol, International Journal of Phytomedicine, 3, 459-469 (2011).

2. A.M. PISOSCHI, A. POP, The role of antioxidants in the chemistry of oxidative stress: a Review, European Journal of Medicinal Chemistry (2015).

3. O.I. ARUOMA, Free Radicals, Oxidative Stress, and Antioxidants in Human Health and Disease, JAOCS, 75(2) (1998). 
4. K. ZHOU, J.J. RAFFOUL, Potential Anticancer Properties of Grape Antioxidants, Journal of Oncology, Volume 2012 (2012).

5. M. RINNERTHALER, J. BISCHOF, M.K. STREUBEL A. TROST, K. RICHTER, Oxidative Stress in Aging Human Skin, Biomolecules, 5, 545-589 (2015).

6. B. DUMITRIU, L. OLARIU, R. NITA, L. ZGLIMBEA N. ROSOIU, Vascular anti-inflammatory effects of natural compounds from Aesculus hippocastanum and Hedera helix, Romanian Biotechnological Letters, 18(1), 7963-7974 (2013).

7. D.G. DEAVALL, E.A. MARTIN, J.M. HORNER, R. ROBERTS, Drug-Induced Oxidative Stress and Toxicity, Journal of Toxicology, 2012 (2012).

8. B. DUMITRIU, L. OLARIU, M.D. ENE, L. ZGLIMBEA, N. ROSOIU, The Photo-Protective Activity of Crataegus monogyna Extract Proved by Complementary Mechanisms of Skin Inflammation, Damage of Extracellular Matrix, Cellular Survival and Proliferation Disorders, International Journal of Biotechnology for Wellness Industries, 1, 177-188 (2012)

9. E. ESPOSITO, S. CUZZOCREA, TNF-alpha as a therapeutic target in inflammatory diseases, ischemiareperfusion injury and trauma, Curr Med Chem., 16(24), 3152-67 (2009).

10. L.R. DeCHATELET, P.S. SHIRLEY, R.B. Jr. JOHNSTON, Effect of phorbol myristate acetate on the oxidative metabolism of human polymorphonuclear leukocytes, Blood., 47(4), 545-54 (1976).

11. M.S. BLOIS, Antioxidant Determinations by the Use of a Stable Free Radical, Nature, 181, 1199-1200 (1958).

12. V. BONDET, W. BRAND-WILLIAMS, C. BERSET, Kinetics and mechanisms of antioxidant activity using the DPPH free radical method, Lebensmitt Wissenschaft Technologie Food Sci Technol, 30, 609-615 (1997).
13. W. BRAND-WILLIAMS, M.E. CUVELIER, C. BERSET, Use of a free radical method to evaluate antioxidant activity, Food Sci Technol 28, 25-30 (1995).

14. O. EREL, A novel automated direct measurement method for total antioxidant capacity using a new generation, more stable ABTS radical cation, Clinical Biochemistry, 37, 277-285 (2004).

15. M. KAMPA, A. NISTIKAKI, V. TSAOUSIS, N. MALIARAKI, G. NOTAS, E. CASTANAS, A new automated method for the determination of the Total Antioxidant Capacity (TAC) of human plasma, based on the crocin bleaching assay, BMC Clinical Pathology, 2 (2002).

16. S. MUELLER, H-D. RIEDEL, W. STREMMEL, Direct Evidence for Catalase as the Predominant $\mathrm{H} 2 \mathrm{O} 2$ -Removing Enzyme in Human Erythrocytes, Blood, 90(12), 4973-4978 (1997).

17. J.M. McCORD, I. Fridovich, J. Biol. Chem. 244, 60496055 (1969).

18. D.W. HEDLEY, S. CHOW, Evaluation of Methods for Measuring Cellular Glutathione Content Using Flow Cytometry, Cytometry, 15, 349-358 (1994).

19. C.QUIJANO, L. CASTRO, G. PELUFFO, V. VALEZ, R. RADI, Enhanced mitochondrial superoxide in hyperglycemic endothelial cells: direct measurements and formation of hydrogen peroxide and peroxynitrite, Am J Physiol Heart Circ Physiol., 293(6), 3404-14 (2007).

20. M. Carini, G. Aldini, E. Bombardelli, P. Morazzoni, R. MaffeiFacino, UVB-induced hemolysis of rat erythrocytes: Protective effect of procyanidins from grape seed, Life Sciences, 67 (15), 1799-1814 (2000).

21. J.M. Robinson, Phagocytic leukocytes and reactive oxygen species, Histochem Cell Biol., 131 (4), 465-469 (2009). 